\title{
Proposing arterial stiffness as an alternative way of assessing risk in transient ischaemic attack (TIA) patients
}

\author{
SORAM RU PATEL, ${ }^{1}$ KHALID ALI, ${ }^{1}$ EVA BUNTING, ${ }^{2}$ EMMA BODENHAM, ${ }^{2}$ JEAN TIMEYIN, ${ }^{2}$ \\ CHAKRAVARTHI RAJKUMAR ${ }^{1}$
}

\begin{abstract}
Background: The $A B C D 2$ score is a clinical tool currently categorising transient ischaemic attack (TIA) patients as low or high risk. Arterial stiffness, a novel physiological parameter predicting future cardiovascular events, has not been explored in TIA populations.

Methods: The following arterial stiffness parameters were measured in TIA patients aged $>\mathbf{4 0}$ years: carotid-femoral pulse wave velocity (C-F PWV), carotid-radial pulse wave velocity (C-R PWV) using Complior ${ }^{\circledR}$ and central and peripheral blood pressure (BP) using Arteriograph ${ }^{\circledast}$. Nocturnal BP dipping profiles were measured using 24-h ambulatory blood pressure monitoring. TIA participants were age- and gendermatched with healthy subjects to compare arterial stiffness and BP profiles.

Results: Twenty-four TIA patients, $\mathbf{5 0} \%$ male, of mean \pm SD age $70 \pm 10.1$ years were recruited to the study. Mean \pm SD C-F PWV was $11.5 \pm 3.18 \mathrm{~m} / \mathrm{s}$ and C-R PWV was $10.8 \pm 1.26 \mathrm{~m} / \mathrm{s}$. Published normative data for healthy age- and gendermatched subjects are C-F PWV $10.9 \mathrm{~m} / \mathrm{s}$ and C-R PWV 10.1 $\mathrm{m} / \mathrm{s}$. Our TIA patients had higher central $(130 \mathrm{mmHg})$ than peripheral BP (128 $\mathrm{mmHg})$ compared with healthy subjects in whom central BP is $10-15 \mathrm{mmHg}$ lower than peripheral BP. Thirty-eight percent of TIA patients lacked nocturnal dipping compared with $19 \%$ of healthy subjects.

Conclusions: In our TIA cohort we found increased arterial stiffness measurements compared to healthy subjects. Br J Diabetes 2016;16:16-19
\end{abstract}

Key words: stroke, TIA, arterial stiffness, blood pressure, ambulatory, dippers

Brighton and Sussex Medical School, Brighton, UK

Brighton and Sussex University Hospitals Trust, Brighton, UK

Address for correspondence: Dr Khalid Ali

Academic Department of Geriatrics, Audrey Emerton Building, Royal

Sussex County Hospital, Brighton BN2 5BE, UK

Tel: +44 (0)1273 523360

E-mail: khalid.ali@bsuh.nhs.uk

http://dx.doi.org/10.15277/bjd.2016.057

\section{Introduction}

The incidence of transient ischaemic attack (TIA) ranges from 0.37 to 1.1 per 1000 per year; $1,27-40 \%$ of patients presenting with an ischaemic stroke have suffered a previous TIA. ${ }^{3}$ The true risk of stroke is up to $10 \%$ at 1 week after a TIA and $17 \%$ at 1 month. ${ }^{4,5} \mathrm{~A}$ recent review and meta-analysis of TIA patients triaged to secondary stroke prevention services indicated that $45 \%$ of patients classified as high risk were found to have stroke mimics such as migraine and epilepsy. ${ }^{6}$

Improved risk stratification in TIA patients may assist in identifying patients with true cerebrovascular disease and at higher risk of subsequent cerebrovascular accidents. At present the ABCD2 risk stratification score is a simple tool used to provide prognostic information in identifying TIA patients with greatest short-term risk of stroke and thus requiring urgent specialist assessment within $24 \mathrm{~h}$. These recommendations are partly influenced by service provision of TIA clinics and their capacity to accommodate patients within $24 \mathrm{~h}$. Practical constraints mean that access to these clinics must ideally be reserved for those at highest risk, as opposed to all comers perceived to have had a TIA which includes a high proportion of stroke mimics. The parameters of the $A B C D 2$ score are based solely on clinical features (age, blood pressure, clinical symptoms of unilateral weakness and speech disturbance, duration and diabetes). Some patients classified as low risk (ABCD2 score $<4$ ) may have significant aetiological risk factors, such as atrial fibrillation, which are not included in the score. $7,8 \mathrm{~A}$ recent meta-analysis and systematic review including 13,766 TIA patients from 29 cohorts showed that the $A B C D 2$ score does not reliably distinguish between stroke mimics and those at high risk ( $A B C D 2$ score $\geq 4$ ) of early stroke. ${ }^{6}$ Over 2000 patients classified as low risk (ABCD2 score $<4)$ did have significant carotid stenosis or atrial fibrillation, which are known to independently increase the risk of subsequent stroke events. One in five patients with carotid stenosis requiring urgent intervention were categorised as low risk. Additionally, the ABCD2 score has low sensitivity and specificity when used in the community and by non-specialists. These reasons highlight the need for improved methods to predict the future risk of strokes in TIA patients.

Novel cardiovascular parameters such as measures of arterial stiffness may potentially provide a more sophisticated risk assessment than the $A B C D 2$ score. ${ }^{9}$ Epidemiological studies have determined that increased arterial stiffness independently predicts cardiovascular events including myocardial infarction and stroke. ${ }^{10-15}$ 
Arterial stiffness can be measured reliably and non-invasively using pulse wave velocity (PWV). ${ }^{16-19}$ Exploring measurements of arterial stiffness in TIA patients specifically may improve the identification of patients at high risk of subsequent stroke events. Peripheral blood pressure (BP) measurements are a component of the $A B C D 2$ scoring system. It must be borne in mind that the interplay of vascular parameters is co-dependent on BP characteristics and the site of $\mathrm{BP}$ measurement. Brachial BP does not accurately represent systemic $B P$, as systolic $B P(S B P)$ is amplified between the aortic root and periphery. Several factors affect the relationship between brachial and aortic BP, such as ageing, diabetes, atherosclerosis and gender. ${ }^{20,21}$

Traditionally, peripheral BP but not central BP is measured in clinical practice. However, measuring central SBP is a useful, reliable indicator of the degree of aortic stiffening compared with peripheral arterial measurements. ${ }^{11,15,22}$ The relationship between central and peripheral BP in TIA patients has not yet been determined.

Twenty-four hour BP can be measured simply and non-invasively in the community setting. BP dips by $10-20 \%$ overnight in healthy individuals. Lack of nocturnal dipping has been associated with worse vascular stiffness parameters and poor outcomes in ischaemic stroke patients after 3 weeks and, similarly, the nocturnal BP dipping phenomenon has not been explored in TIA patients. ${ }^{23-25}$

This study aims to measure vascular parameters (arterial stiffness and BP dipping profile) in a cohort of TIA patients and compare their PWVs and BP characteristics with published values for healthy individuals from an existing database.

The primary hypothesis is that TIA patients have stiffer arteries than subjects who have not suffered a TIA. Secondary hypotheses are that (1) TIA patients have a higher central BP than peripheral BP compared with healthy subjects, suggesting stiffer aorta; and (2) TIA patients are more likely to lack nocturnal dipping in 24-h ambulatory blood pressure monitoring (ABPM) than healthy subjects.

\section{Methods}

Participants were recruited from the Brighton and Sussex University Hospitals Trust (BSUH) TIA Rapid-Access clinic and stroke ward. Inclusion criteria: participants aged $\geq 40$ years with a confirmed diagnosis of TIA. Exclusion criteria: participants in atrial fibrillation, malignancy with current active treatment, dementia or previous TIA. Atrial fibrillation confounds arterial stiffness measurements, so these participants were excluded.

TIA diagnoses were confirmed by the stroke physician following an assessment including history, examination and investigations (e.g CT head, MRI scan, ECG and carotid Doppler).

Participants underwent baseline assessment including a full medical history and examination. Body mass index $\left(\mathrm{kg} / \mathrm{m}^{2}\right)$ was calculated. Lying and standing BP and heart rate were measured using a validated oscillometric device (Omron HEN-705CP, Tokyo, Japan) at the right brachial artery.

PWV was measured in the carotid-femoral C-F (elastic) and carotid-radial C-R (muscular) arterial segments using Complior ${ }^{\circledR}$ (Alam Medocas, France), a validated automated system to measure arterial stiffness.
Central aortic BP was measured non-invasively using oscillometric Arteriograph ${ }^{\circledR}$ (TensioClinic Arteriograph; UniMedic) equipment. Participants were positioned at $45^{\circ}$ recline and a BP cuff was placed. The measurements were all performed by the same two researchers.

Repeatability calculations indicated minimal bias from intraand inter-observer variability (intra-observer repeatability coefficient (RC); C-F PWV $=4.3 \%, C-R$ PWV $=5.5 \%$; inter-observer RC; C-F PWV $=2.3 \%, C-R P W V=4.1 \%$ ).

Twenty-four hour ABPM was measured using Diasys Integrall machines (Novacor, Cedex, France). BP was measured every 30 min between 07:00 and 22:00 (day), and hourly between 22:01 and 06:59 (night) over $24 \mathrm{~h}$. A cut-off of five valid overnight recordings was used. The results for each participant were classified according to diurnal SBP variation (SBP change $(\%)=($ day SBP - night SBP)/day SBP) as dippers ( $\geq 10 \%$ fall in BP overnight), non-dippers ( $0-<10 \%$ fall in $B P$ overnight) and reverse dippers $(>0 \%$ rise in BP overnight).

Twenty-four hour BP dipping profiles were compared between TIA participants matched for age (within \pm 5 years) and gender with healthy Caucasian subjects from the UK arm of the Brighton and Verona Seniors' Study (BRAVES): Prevalence of unrecognised metabolic syndrome in the healthy elderly population. ${ }^{26}$ In this study 90 healthy subjects aged between 65 and 85 years were determined by selection from a UK GP register. Subjects with known hypertension, diabetes, taking statin therapy or clinically obese were excluded. Comparisons were drawn between ABPM recordings that were measured using the same methodology and techniques in both the BRAVES study and our TIA cohort. Ethical approval was obtained from Brighton Research Ethics Committee (REC number: 09/H1107/86).

\section{Results}

Twenty-six participants were initially recruited. One patient was subsequently found to be in atrial fibrillation and the ABPM result for one patient was incomplete. Thus data from 24 participants were analysed; 22 had valid PWV recordings, of which 16 had valid ABPM recordings. The mean $\pm S D$ age of the participants was $70 \pm 10.1$ years (range $50-86$ ) and $50 \%$ were male. Table 1 summarises the baseline characteristics of the cohort.

\section{PWV}

PWV recorded in different segments are summarised in Table 2. Twenty-two TIA participants had valid C-F PWV and C-R PWV recordings.

\section{Central vs brachial SBP}

Central SBP was higher than peripheral SBP in TIA participants (central SBP $=130 \pm 19.3 \mathrm{mmHg}$, brachial SBP $=128 \pm 14.7 \mathrm{mmHg}$, $n=20$ ), with a mean difference of $2 \mathrm{mmHg}$ between the two measurements. In healthy populations one would normally expect a lower central BP compared with the brachial BP.

\section{Nocturnal dipping}

From this TIA cohort, 16 participants had valid ABPM recordings 
Table 1 Characteristics of TIA participants $(n=24)$ showing the mean \pm SD (range) for all variables

\begin{tabular}{llll}
\hline & Mean & SD & Range \\
Age (yrs) & 70 & \pm 10.1 & $(50-86)$ \\
Body mass index $\left(\mathbf{k g} / \mathbf{m}^{2}\right)$ & 28.5 & \pm 6.50 & $(20.8-41.4)$ \\
Smoking (pack years) & 27 & \pm 35.9 & $(0-150)$ \\
Alcohol (units/week) & 6 & \pm 9.5 & $(0-40)$ \\
Total cholesterol (mmol/L) & 5.1 & \pm 1.32 & $(2-8)$ \\
SBP (mmHg) & 138 & \pm 19.0 & $(98-175)$ \\
DBP (mmHg) & 80 & \pm 9.9 & $(55-95)$ \\
MAP (mmHg) & 99 & \pm 10.9 & $(73-120)$
\end{tabular}

Table 2 Comparison between PWV values recorded in this TIA cohort compared with published normal PWV values

\begin{tabular}{llll}
\hline & Technique & $\begin{array}{l}\text { TIA participants } \\
\pm \text { SD }(\mathbf{m} / \mathbf{s})\end{array}$ & $\begin{array}{l}\text { Normal healthy } \\
(\mathbf{m} / \mathbf{s}) \text { Study }\end{array}$ \\
$\begin{array}{l}\text { C-F PWV } \\
(\mathrm{n}=22)\end{array}$ & Complior $^{\circledR}$ & 11.5 & 10.9 \\
& & \pm 3.18 & $\begin{array}{l}\text { Mattace-Raso et al, } \\
2010^{27}\end{array}$ \\
$\begin{array}{l}\text { C-R PWV } \\
\text { (n=22) }\end{array}$ & Complior $^{\circledR}$ & 10.8 & 10.1 \\
& & \pm 1.26 & Ramsay et al, $2012^{26}$
\end{tabular}

Figure 1. Difference in frequencies of types of dippers between TIA cohort (dark blue, $\mathrm{n}=16$ ) and healthy subjects (light blue, $n=16)(p=0.232)$

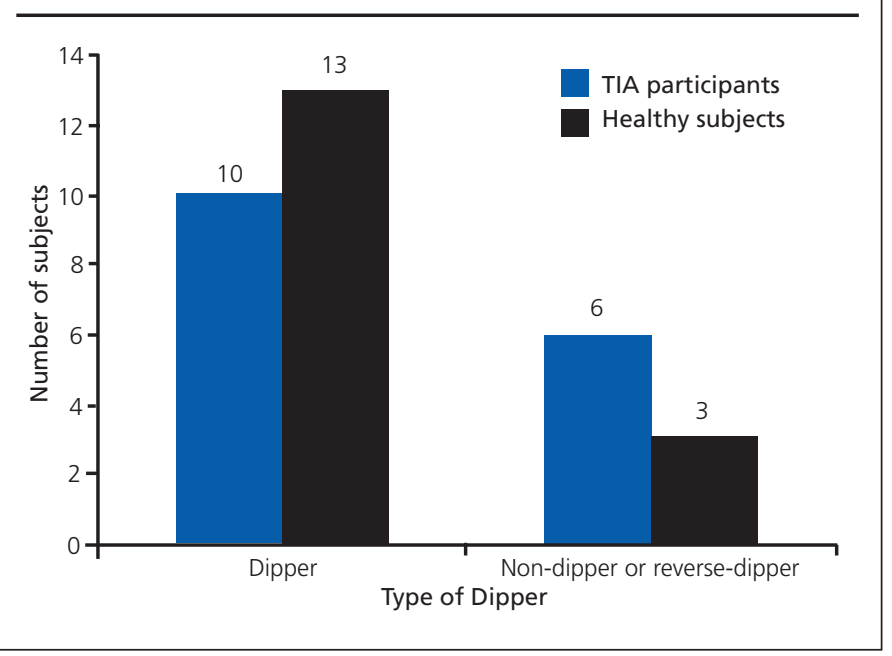

and were matched for gender and age ( \pm 5 years) to healthy subjects (Figure 1). Six TIA participants (38\%) lacked nocturnal dipping compared with $19 \%$ of matched healthy subjects, five of whom were non-dippers and one was a reverse-dipper. SBP dipped nocturnally in 10 patients (62\%).

\section{Discussion}

The key findings of our data suggest that participants who have suffered a TIA have stiffer arteries compared with published normative data (Table 2). Our TIA cohort had a C-F PWV $0.6 \mathrm{~m} / \mathrm{s}$ higher than healthy subjects in published data and a C-R PWV $0.7 \mathrm{~m} / \mathrm{s}$ higher than healthy subjects in published data. ${ }^{26,27}$ This finding of increased arterial stiffness has also been associated with a greater central aortic BP compared to peripheral brachial BP (central SBP = $130 \mathrm{mmHg}$, peripheral SBP $=128 \mathrm{mmHg}$ ), and a lower prevalence of nocturnal dipping in SBP than in healthy subjects (TIA nondippers or reverse dippers $=38 \%$, matched healthy non-dippers or reverse dippers $=19 \%, p=0.232(\mathrm{n} / \mathrm{s}))$.

Central SBP was higher by $2 \mathrm{mmHg}$ than peripheral SBP in individuals who have suffered a TIA. Peripheral SBP is normally amplified from the aorta. ${ }^{20}$ Central BP is expected to be $10-15$ $\mathrm{mmHg}$ lower than peripheral BP in healthy patients. ${ }^{28}$ The role of central BP is not yet established in the aetiology of TIA. Our findings are important as central BP can be measured non-invasively in clinical environments and may be a characteristic factor for some TIA patients. Thus, further investigation of this proposed relationship may lead to improved identification of future TIA risk compared with the ABCD2 scoring system.

The percentages of non-dippers in the TIA cohort (38\%) was greater than that reported in a group of healthy elderly patients (14\%). ${ }^{29}$ Thirty-eight percent of TIA participants compared with $19 \%$ of matched control subjects lacked nocturnal dipping. The likelihood of a TIA participant, compared to a healthy subject, being a non-dipper was not statistically significant. A larger sample of TIA patients showing a non-dipping BP profile is needed to explore the association between non-dipping BP and future risk of TIA and stroke events. It is physiologically plausible that patients with non-dipping BP profiles may be at higher risk of atherosclerosis, worse vascular parameters and higher risk of future stroke events.

The ABCD2 scoring system is open to recall bias, does not include significant risk factors such as carotid stenosis and atrial fibrillation and, as such, has limited risk prediction properties due to the heterogeneity of TIA patients. ${ }^{7,8}$ The measures of direct arterial stiffness and blood pressure parameters (central BP and dipping profile) proposed in the study reflect biological age (rather than chronological age), and hence may offer a more objective and relevant risk prediction than the existing $A B C D 2$ risk stratification system.

\section{Study limitations}

TIA participants were retrospectively matched to healthy subjects. Although participants were age- and gender-matched and those with atrial fibrillation excluded, risk factors such as diabetes, cholesterol, alcohol and smoking were not matched for. ${ }^{30}$

\section{Conclusions}

From our TIA cohort we found that TIA participants have stiffer arteries in the majority of arterial segments compared with a database of healthy subjects. TIA participants have stiffer aortas as determined by PWV, central BP being greater than peripheral 


\section{Key messages}

- $\quad V A B C D 2$ risk scoring system does not reliably differentiate between TIAs and stroke mimics

- The ABCD2 scoring system does not take into account significant vascular risk parameters such as arterial stiffness and dipping blood pressure profile

- Our cohort of TIA patients had increased arterial stiffness measurements compared to age and gender matched healthy older adults

- We advocate exploring the potential of arterial stiffness and blood pressure dipping profile as potentially objective vascular parameters in future risk prediction of TIAs and stroke

$\mathrm{BP}$ and a higher proportion of non-dippers or reverse dippers. These vascular parameters are not currently included in risk stratification scores such as the $A B C D 2$. This study advocates exploring their potential as more objective vascular parameters in TIA patients.

\section{Conflict of interest None}

Funding No external funding was secured but the study was sponsored by the R\&D department at Brighton and Sussex University Hospitals NHS Trust.

\section{References}

1. Truelsen T, Begg S, Mathers $C$. The global burden of cerebrovascular disease. Geneva: World Health Organisation; 2000.

2. Hippisley-Cox J, Pringle M, Ryan R. Stroke: prevalence, incidence and care in general practices 2002 to 2004. Final report to the national stroke audit team. London: Royal College of Physicians; 2004.

3. Easton JD, Saver JL, Albers GW, et al. Definition and evaluation of transient ischemic attack: a scientific statement. Stroke 2009;40(6):227693. http://dx.doi.org/10.1161/STROKEAHA.108.192218

4. Rothwell PM, Warlow CP. Timing of TIAs preceding stroke: time window for prevention is very short. Neurology 2005;64:817-20. http://dx.doi.org/10.1212/01.WNL.0000152985.32732.EE

5. vanWijk I, Kappelle LJ, van Gijn J, et al. Long-term survival and vascular event risk after transient ischaemic attack or minor ischaemic stroke: a cohort study. Lancet 2005;365(9477):2098-104

http://dx.doi.org/10.1016/S0140-6736(05)66734-7

6. Wardlaw JM, Brazzelli M, Chappell FM, et al. ABCD2 score and secondary stroke prevention: meta-analysis and effect per 1000 patients triaged. Neurology 2015;85(4):373-80.

http://dx.doi.org/10.1212NWL.0000000000001780

7. Johnston SC, Rothwell PM, Huynh-Huynh MN, et al. Validation and refinement of scores to predict very early stroke risk after transient ischemic attack. Lancet 2007;369:283-92.

http://dx.doi.org/10.1016/S0140-6736(07)60150-0

8. Bhatt $A$, Jani $V$. The $A B C D$ and $A B C D 2$ scores and the risk of stroke following a TIA: a narrative review. ISRN Neurol 2011:518621. http://dx.doi.org/10.5402/2011/518621

9. Rothwell PM. Lack of epidemiological data on secondary stroke prevention. Lancet 2005;4(9):518-19. http://dx.doi.org/10.1016/S1474-4422(05)70148-4

10. Laurent $S$, Boutouyrie $P$, Asmar $R$, et al. Aortic stiffness is an independent predictor of all-cause and cardiovascular mortality in hypertensive patients. Hypertension 2001;37(5):1236-41. http://dx.doi.org/10.1161/01.HYP.37.5.1236

11. Mitchell GF, Hwang SJ, Vasan RS, et al. Arterial stiffness and cardiovascular events: the Framingham heart study. Circulation 2010;121(4):505-
11. http://dx.doi.org/10.1161/CIRCULATIONAHA.109.886655

12. Boutouyrie $\mathrm{P}$, Tropeano Al, Asmar R, et al. Aortic stiffness is an independent predictor of primary coronary events in hypertensive patients: a longitudinal study. Hypertension 2002;39(1):10-5. http://dx.doi.org/10.1161/hy0102.099031

13. Maldonado J, Pereira T, Polonia J, et al. Arterial stiffness predicts cardiovascular outcome in a low-to-moderate cardiovascular risk population: the EDIVA (estudo de distensibilidade vascular) project. I Hypertens 2011;29(4):669-75. http://dx.doi.org/10.1097/HJH.0b013e3283432063

14. Mattace-Raso FU, van der Cammen TJ, Hofman A, et al. Arterial stiffness and risk of coronary heart disease and stroke: the Rotterdam study. Circulation 2006;113(5):657-63. http://dx.doi.org/10.1161/CIRCULATIONAHA.105.555235

15. Kingwell BA, Waddell TK, Medley TL, et al. Large artery stiffness predicts ischemic threshold in patients with coronary artery disease. J Am Coll Cardiol 2002;40(4):773-9. http://dx. doi.org/10.1016/S0735-1097(02)02009-0

16. Wilkinson IB, McEniery CM, Cockcroft JR. Pulse waveform analysis and arterial stiffness: realism can replace evangelism and scepticism. $J$ Hypertens 2005;23(1):213. http://dx.doi.org/10.1097/00004872-200501000-00032

17. Gladdish S, Manawadu D, Banya W, et al. Repeatability of non-invasive measurement of intracerebral pulse wave velocity using transcranial Doppler. Clin Sci (Lond) 2005;108(5):433-9. http://dx.doi.org/10.1042/CS20040251

18. Laurent S, Cockcroft J, Van Bortel L, et al. Expert consensus document on arterial stiffness: methodological issues and clinical outcomes. Eur Heart $J$ 2006;27(21):2588-605. http://dx.doi.org/10.1093/eurheartj/eh|254

19. Scuteri A, Sgorbini L, Leggio F, Brancati AM. Aortic correlates of clinical markers of large artery structure and function. Aging Clin Exp Res 2006;18(5):452-61. http://dx.doi.org/10.1007/BF03324843

20. Karamanoglu M, O'Rourke MF, Avolio AP, Kelly RP. An analysis of the relationship between central aortic and peripheral upper limb pressure waves in man. Eur Heart J 1993;14(2):160-7. http://dx.doi.org/10.1093/eurheartj/14.2.160

21. McEniery CM, Yasmin, McDonnell B, et al. Central pressure: variability and impact of cardiovascular risk factors: the Anglo-Cardiff Collaborative Trial II. Hypertension 2008;51(6):1476-82. http://dx.doi.org/10.1161/HYPERTENSIONAHA.107.105445

22. Roman MJ, Devereux RB, Kizer JR, et al. Central pressure more strongly relates to vascular disease and outcome than does brachial pressure: the strong heart study. Hypertension 2007;50(1):197-203.

http://dx.doi.org/10.1161/HYPERTENSIONAHA.107.089078

23. Li Y, Wang JG, Dolan E, et al. Ambulatory arterial stiffness index derived from 24-hour ambulatory blood pressure monitoring. Hypertension 2006; 47(3):359-64. http://dx.doi.org/10.1161/01.HYP.0000200695.34024.4c

24. Dolan E, Li Y, Dolan E, et al. Ambulatory arterial stiffness index as a predictor of cardiovascular mortality in the Dublin Outcome Study. Hypertension 2006;47(3):365-70. http://dx.doi.org/10.1161/01.HYP.0000200699.74641.c5

25. Sturrock ND, George E, Pound N, et al. Non-dipping circadian blood pressure and renal impairment are associated with increased mortality in diabetes mellitus. Diabet Med 2000;17(5):360-4. http://dx.doi.org/10.1046/j.1464-5491.2000.00284.x

26. Ramsay I, Fantin F, Zamboni M, et al. Prevalence of unrecognised metabolic syndrome in the healthy elderly population-Brighton and Verona seniors study (BRAVES). Age Ageing 2013;42(s2):ii26-27.

27. Mattace-Raso F, Hofman A, Verwoert $G$, et al. Determinants of pulse wave velocity in healthy people and in the presence of cardiovascular risk factors: 'establishing normal and reference values'. Eur Heart $J$ 2010;31(19):2338-50. http://dx. doi.org/10.1093/eurheartj/ehq165

28. Williams B, Lacy PS, Thom SM, et al. Differential impact of blood pressure-lowering drugs on central aortic pressure and clinical outcomes: principal results of the conduit artery function evaluation (CAFE) study. Circulation 2006;113(9):1213-25.

http://dx.doi.org/10.1161/CIRCULATIONAHA.105.595496

29. O'Brien E. Ambulatory blood pressure monitoring in the management of hypertension. Heart 2003;89(5):571-6. http://dx.doi.org/10.1136/heart.89.5.571

30. Sacco RL, Benjamin EJ, Broderick JP, et al. American Heart Association Prevention Conference IV. Prevention and Rehabilitation of Stroke. Risk factors. Stroke 1997;28(7):1507-17. 